\title{
LAS RAÍCES EPISTEMOLÓGICAS DEL REALISMO ESTRUCTURAL: MODELOS Y REPRESENTACIÓN EN LA TEORÍA DE RELACIONES INTERNACIONALES
}

\author{
THE EPISTEMOLOGICAL ROOTS OF STRUCTURAL REALISM: MODELS AND \\ REPRESENTATION IN INTERNATIONAL RELATIONS THEORY
}

Juan Francisco Morales Giraldo *

\section{RESUMEN}

El texto explora algunas ideas básicas acerca de la epistemología detrás de la teoría del Realismo Estructural, o Neorrealismo, de Kenneth Waltz. En primer lugar, se aborda el problema de la interpretación descriptiva de las teorías, particularmente en la disciplina de Relaciones Internacionales, así como ciertas concepciones críticas que surgen desde esta lógica. En segundo lugar, se analiza la estructura formal de los programas teóricos en general, con énfasis en la estructura teórica del Realismo Estructural. La noción de la dualidad de los fenómenos políticos internacionales es descrita como un elemento central en el núcleo de leyes y principios empíricos de la teoría, definiendo su propósito y estableciendo sus alcances y limitaciones. Finalmente, la última sección explora el potencial predictivo de las teorías de relaciones internacionales, elaborando un planteamiento crítico sobre estos temas desde la propia teoría. Finalmente, las conclusiones ponen en consideración la contribución de la teoría general de Waltz sobre el modo de pensar las teorías y su importancia para la disciplina de las Relaciones Internacionales.

Palabras clave: Realismo Estructural. Teorías Empíricas. Modelos Teóricos. Concepción Semántica.

\begin{abstract}
This text addresses some basic ideas about the epistemology behind Kenneth Waltz's Structural Realism or Neorealism. First, the article deals with the problem of the descriptive interpretation of theories, particularly in International Relations studies, and certain criticism conceptions that comes from this particular logic. The next section presents an analysis of the formal structure of theory programs in general, with an emphasis on theoretical structure of Structural Realism. The notion of duality of international political phenomenons is identified as a central element in the core of empirical laws and principles of this theory, defining its purpose and stablishing its scope and limitations. Finally, the last part focuses on the potential of International Relations theories for predictions, and elaborates a critical approach from the theory to these aspects. Finally, the conclusions put on consideration the contributions of Waltz's

\footnotetext{
* Maestro en Sociología en Estudios Políticos por la Universidad Nacional Mayor de San Marcos (UNMSM). Licenciado en Ciencia Política por la Pontificia Universidad Católica del Perú (PUCP). Es investigador del Instituto de Estudios Políticos Andinos (IEPA) y del Grupo de Trabajo "Integración y Unidad Latinoamericana" del Consejo Latinoamericano de Ciencias Sociales (Clacso). Ha desempeñado labores de docencia en la UNMSM y en la PUCP. Contacto: jfmoralesg.88@gmail.com
} 
general theory on how we think about theories and its importance for International Relations discipline.

Keywords: Structural Realism. Empirical Theories. Theoretical Models. Semantic View.

\section{Introducción}

El Realismo Estructural, o Neorrealismo', ha sido la teoría de relaciones internacionales más influyente desde su aparición a finales de la década de 1970. Su contribución a la disciplina puede evaluarse desde muchos aspectos, pero uno de los más importantes es quizás el menos discutido en la literatura especializada y en los programas de enseñanza: no sólo es una teoría sobre política internacional, también representa un compromiso filosófico que ha buscado acercar el estudio científico de las relaciones internacionales a los principios generales que rigen el quehacer de las ciencias empíricas. En ese sentido, los aportes del estructuralismo de Waltz van más allá de la comprensión de un dominio particular de hechos; ofrece una primera aproximación a la importancia del trabajo teórico en la ciencia política. La discusión sobre temas fundamentales acerca de qué son las teorías en un sentido epistemológico, cómo funcionan y cómo se construyen, contenidos en el primer capítulo del clásico Theory of International Politics (1979), conformaban una aproximación crítica al debate filosófico acerca de la naturaleza de las teorías científicas, su relación con la realidad empírica, y su rol en la ciencia social moderna.

El texto que sigue a continuación es una mirada a algunos elementos epistemológicos sobre los cuales se funda el Realismo Estructural. Los apartados se dividen en tres temáticas generales: la descripción, la explicación y la predicción de las teorías científicas, a lo largo de las cuales se establecerán paralelos con algunos temas centrales para las ciencias empíricas, y se plantearán discusiones en torno a los tipos de problemas que aborda la propia teoría. Estos paralelos y discusiones servirán tanto para ilustrar los temas a tratar, así como para introducir una selección bibliográfica especializada que orienten al lector hacia un tratamiento más detallado de estas cuestiones $^{2}$.

\footnotetext{
${ }^{1}$ Ambos términos, Realismo Estructural y Neorrealismo, son útiles en la medida en que resaltan algún aspecto sustancial de la teoría: el primero, señalara Robert Keohane, “. . . capta la atención prioritaria en la explicación a través de un examen de la estructura del sistema internacional" (1993, 68, nota 17); el segundo, capta mejor su estatus fundacional dentro de la corriente realista y su originalidad en la teoría política internacional. Para una revisión temática y bibliográfica de las principales vertientes neorrealistas hasta la fecha, a modo de recuento, véase Lobell (2010). Para una revisión de la tradición realista en general, junto con una selección bibliográfica temática, véase Viotti y Kauppi (2012, cap. 2).

${ }_{2}^{2}$ Para facilitar la revisión de las referencias a Theory of International Politics, se incluirán también los números de página correspondientes a su edición en castellano, separadas de la numeración de la edición en inglés por una diagonal (/).
} 


\section{Teorías: descripción, modelos y representación}

Existen dos puntos de vista desde los cuales las teorías científicas podrían considerarse descriptivas en un sentido formal. En primer lugar, pueden ser descriptivas en el sentido de hacer explícitas las propiedades y relaciones que subyacen a cierta clase de hechos (en este caso, fenómenos y procesos internacionales) y que sirven a su comprensión. Este tipo de descripción es común a todas las ramas de la ciencia empírica. “... Una teoría científica -decía el físico Stephen Hawking- es tan sólo un modelo matemático que hacemos para describir nuestras observaciones [...] Sólo existe en nuestra mente" (Hawking, 1999, p. 106). Aquí hay dos importantes pistas acerca de lo que debe entenderse por una teoría científica: las teorías son modelos de la realidad, son construcciones intelectuales, no son descripciones exhaustivas ni una colección de datos observacionales. Las teorías no detallan el mundo tal cual es, sino que recurren a representaciones conceptuales simplificadas de fenómenos y procesos. En filosofía de la ciencia, son estudiadas como modelos teoréticos: "sistemas hipotético-deductivos concernientes a un objeto modelo que es, a su vez, una representación conceptual esquemática de un fenómeno real" (Bunge, 1972, pp. 15-16). En la disciplina de las Relaciones Internacionales, estos modelos teoréticos, comúnmente integrados bajo el marco de teorías generales y que abarcan teorías específicas, especializadas, o auxiliares $^{3}$, se construyen siempre en referencia a la problemática de la internacionalidad ${ }^{4}$.

Hasta la década de 1970, la filosofía de la ciencia estaba dominada por la llamada Concepción Heredada, un enfoque según el cual éstas constituyen sistemas de axiomas empíricamente interpretados ${ }^{5}$. Luego del periodo historicista dominante hasta la década de 1980, la llamada Concepción Semántica cobró fuerza como la corriente dominante en la filosofía de la ciencia. La nueva perspectiva semántica entiende las teorías como entidades modelo-teóricas: presentar una teoría es presentar una clase de modelos (Díez, 1997, pp. 42, 46), que en su acepción no formal, son de naturaleza representacional: son representaciones conceptuales $-\mathrm{O}$ simplemente conceptualizaciones- de ciertos aspectos del mundo empírico (Falguera, 1992, p. 100) ${ }^{6}$.

La relación entre teoría y modelo está dada, en parte, por una de las principales características de la ciencia empírica: su vocación analítica. "La investigación comienza

\footnotetext{
${ }^{3}$ Sobre teorías generales y modelos teóricos, véase Bunge (1982, p. 181)

4 "El conjunto de los fenómenos sociales que expresan relaciones inmediatas de los Estados entre sí o influyen directa o indirectamente en estas relaciones o están por ellas influidos, constituye el problema de la internacionalidad. Es internacional una relación cuando se refiere a las relaciones entre grupos sociales que están determinados por poderes estatales distintos..." (Huber, citado en Del Arenal, 2007, p. 405).

${ }^{5}$ Sobre esto, véase Díez y Moulines (1997, cap. 8).

${ }^{6}$ Desde la década de 1960, los proponentes de la concepción semántica de las teorías científicas han venido discutiendo acerca de la existencia de un significado común de la noción de modelo que subyace a todos sus demás usos en las ciencias formales y en las ciencias empíricas. Bajo esos diferentes usos del término modelo, dice el filósofo español José Luis Falguera, “... subyace una condición común; de manera que dichos usos resultan de condiciones específicas que adopta una noción común de 'modelo', conforme a diferentes maneras de darse la relación de 'ser modelo de'. Según esto, algo es modelo, para cualquier de los usos señalados, en tanto que es un sistema mediante el que se postula una representación conceptual de un asunto determinado - real o ficticio- conforme a determinada finalidad, constituyendo tal representación conceptual un sistema abstracto. Así pues, conforme a esto, el principal papel de los modelos es el de representar" (Falguera, 1993, pp. 173-174; énfasis en el original). Véase también, Falguera (1994).
} 
descomponiendo sus objetos a fin de descubrir el 'mecanismo' interno responsable de los fenómenos observados" - dice el filósofo Mario Bunge-; y una vez investigada la naturaleza de las partes de ese mecanismo subyacente, el siguiente paso es el examen de la interdependencia de esas partes, y finalmente, la reconstrucción del todo en términos de sus partes interconectadas (Bunge, 1997a, p. 26). En un plano muy general, respecto a ciertos ámbitos o sistemas de la realidad, esto ocurre por medio de teorías. "... Los científicos tratan de urdir modelos teóricos que representen los principales rasgos de los sistemas de interés (Bunge, 1999, p. 236). De esa manera, a) las teorías describen elementos que están más allá de las apariencias; $b$ ) describen una clase de hechos y no hechos aislados; fijan su atención en hechos genéricos (abstracciones que en ciencias sociales llamamos "hechos sociales"), no en hechos singulares, con lo cual $c$ ) rechazan los aspectos puramente accidentales de la realidad, seleccionando sólo los que se consideran más relevantes para los propósitos de la investigación. El conocimiento científico, entonces, a través de la teorización, trasciende las contingencias de la percepción inmediata, "... racionaliza la experiencia en lugar de limitarse a describirla...". El investigador conjetura acerca de lo que subyace tras los fenómenos observados, busca establecer pautas regulares que los expliquen, y busca establecer mediante esta comprensión cierto grado de predictibilidad. Emplean conceptos — por ejemplo, adaptación, integración, clase social o tendencia histórica- que carecen de un correlato empírico por no corresponden a fenómenos directamente observables, pero que sí se refieren a hechos, cualidades o relaciones generales existentes objetivamente (Bunge, 1997a, p. 24). Las teorías, en resumen, son deconstrucciones selectivas de la realidad. No describen exhaustivamente lo observado, sino lo que subyace a los hechos. La ciencia, al ser analítica y explicativa, es en cierto sentido esencialista: “... intenta llegar a la raíz de las cosas. Encuentra la esencia [de los fenómenos] en variables pertinentes y en las relaciones invariantes entre ellas" (Bunge, 1997a, p. 38).

\section{El problema de la interpretación descriptiva de las teorías}

El Realismo Estructural parte de una base filosófica cercana a una concepción semántica de las teorías científicas, cuyos planteamientos más básicos respecto al rol de los modelos han sido ya expuestos de manera sucinta en la sección anterior. "Una teoría es una imagen, construida mentalmente, de cierto ámbito o dominio de actividad limitado -en este caso, de un cierto ámbito de las relaciones sociales: la política internacional-. Una teoría es una representación de la organización de ese dominio y de las relaciones entre sus partes" (Waltz, 1979, p. 8/19; 1990, p. 26). Aunque Waltz no se adhiere de manera explícita a ninguna escuela filosófica en particular, la idea de teoría que subyace a su obra es la de sistemas representacionales, y su construcción, una tarea fundamentalmente creativa (Waltz, 1979, p. 11/23). Las teorías, por lo tanto, no son una colección de enunciados descriptivos. Lo visto en la sección anterior amplía los alcances de esta concepción filosófica, estableciendo paralelos con las bases epistemológicas del estructuralismo internacional. Los elementos meta teóricos en el primer capítulo de Theory of International Politics condensan los aportes de diferentes ramas de la filosofía de la ciencia, apelando a la unidad de los rasgos característicos de la ciencia moderna ${ }^{7}$. La bibliografía citada a lo largo de este trabajo busca dar mayor

\footnotetext{
7 Al sugerir, por ejemplo, que las teorías, en lugar de ser un conjunto de leyes, las explican, Waltz reconoce lo siguiente: "Este significado no está de acuerdo con el uso que se le da en gran parte de la teoría política internacional, preocupada más por la interpretación filosófica que por la explicación
} 
alcance a conceptos fundamentales tales como "imagen" o "representación", y al hecho más simple de que de las teorías son abstracciones representativas modeladas por medio de conceptos y enunciados lógicos (formales) que los articulan.

Hasta la década de 1960, los autores realistas no habían logrado prescindir del conductismo, del análisis de la política exterior, y el interés por la diplomacia, como enfoques para una aproximación a los asuntos internacionales. Otros, incluso, se mostraban escépticos acerca de la posibilidad de desarrollar una teoría de relaciones internacionales. Un prominente internacionalista británico de la época titulaba un artículo con una inquietante interrogante: Why is there no International Theory? "¿Por qué no existe una teoría de relaciones internacionales?", Wight, 1966). La epistemología con la que Theory of International Politics buscaba introducir nuevos modos de teorización en este campo entraron en marcado contraste con este escepticismo. La distancia frente a trabajos clásicos como el Raymond Aron, Hans Morgenthau, o el propio Martin Wight, se hizo notoria, particularmente en revisiones críticas como el más reciente Realist Though and Neorealist Theory (1990). Por primera vez, entonces, eran abordados aspectos esenciales para una ciencia social de las relaciones internacionales: qué es una teoría, cómo funcionan, y cómo se construyen. El éxito del Realismo Estructural como un programa de investigación progresivo (véase Keohane, 1993, pp. 60-61, 65-97) ${ }^{8}$ supuso desde entonces un nuevo umbral de rigurosidad científica para las nuevas teorías internacionales, no tanto por su capacidad para abordar hechos particulares, o prever situaciones novedosas, sino por la solidez de los principios epistemológicos sobre los que se construyó. El reciente criterio introducido por Waltz para la evaluación teórica exigía a los investigadores examinar el significado de la noción de teoría antes de tratar sobre alguna teoría en concreto ${ }^{9}$.

Frente a esta postura filosófica, sin embargo, tanto críticos como proponentes del Realismo Estructural simplemente dan por sentado que ésta es una teoría sobre la estructura del sistema político internacional, sin prestar atención a la estructura de la propia teoría (cf. Wæver, 2009). Cuando esto es llevado al extremo surge lo que podría llamarse el problema de la interpretación descriptiva de las teorías: un sesgo que expresa la creencia de que los elementos de una teoría son verdaderos o falsos respecto a lo observado porque poseen un correlato empírico directo al que deben ajustarse con precisión y según los cuales deben ser interpretados. En ese sentido, según esta concepción, anclada en los rigores del empirismo lógico, o mejor, en un empirismo

teórica. Corresponde a la definición que se le da al término en las ciencias naturales y en algunas ciencias sociales..." (1979, p. 6/16)

${ }^{8}$ El carácter progresivo de la teoría neorrealista ha sido objeto de debate entre quienes valoran su pertinencia y utilidad. Para apreciaciones críticas en ese aspecto, véanse Vasquez (1997) y Ashley (1986). Las respuestas por parte de prominentes teóricos realistas a las críticas tanto de John Vásquez como de Richard Ashley incidieron en las inexactitudes de su entendimiento acerca del rol las teorías en general y el análisis realista en particular (Waltz, 1997, 1986; Walt, 1997; Gilpin, 1986). Para una defensa del paradigma realista como un programa progresivo, ver también Buzan (1996) y Jervis (2003). Sobre el concepto de progresión teórica, ver Lakatos (1989, pp. 65-72), junto con el resumen de Elman y Elman (2003), desde la concepción historicista, y el detallado recuento de Diez y Moulines (1997, pp. 484-488) desde la concepción estructuralista.

${ }^{9}$ La concepción sintáctica y el historicismo, que sirvieron de soporte para el Realismo Estructural en la década de 1970, introdujeron en la teoría una marcada orientación en contra del positivismo, según el cual las teorías emergen de los hechos observados y éstos son, a su vez, los árbitros de su validez. Para una crítica al inductivismo y el empirismo en la obra de su autor, véase Waltz (2003) y Waever (2009, pp. 203-204). 
exacerbado ${ }^{10}$, los aspectos del mundo empírico y los términos que constituyen una teoría deberían mantener una relación de equivalencia e igualdad. Resultado: las teorías son interpretadas casi literalmente. Pero, ¿es así como procede la ciencia realmente? En una cita en la sección anterior, Stephen Hawking acepta que los modelos matemáticos de los que se sirve la física sólo existen "en la mente de los científicos". Al tratar sobre nociones como tiempo real o tiempo imaginario, por ejemplo, el autor aclara que no tendría sentido preguntarse cuál es más real pues lo importante es que se trata de conceptos que pueden ser más o menos útiles para describir y analizar lo observado (Hawking, 1999, p. 107). Los modelos teóricos y los modelos conceptuales que los conforman, son justamente representaciones de lo observado. En cuanto a la teoría de las relaciones internacionales, algunos ejemplos a continuación ilustrarán esta visión particular de las teorías, y la manera como el Realismo Estructural ha lidiado con el problema del descriptivismo.

Piénsese en la crítica al llamado paradigma Estado-céntrico, que cobró fuerza con el auge del transnacionalismo en la década de 1970. En este paradigma se asumen dos cosas: que los Estados son los actores más importantes de la escena política internacional, y que éstos actúan coherentemente como unidades. El resultado es una suerte de imagen de las relaciones internacionales vistas principalmente como relaciones entre Estados. Pero, ¿qué ocurre con los demás actores y sus relaciones? Si los efectos de las relaciones transnacionales fueran insignificantes y transitorios, señalan los proponentes del transnacionalismo, relegarlos a un rol menor y en general ignorarlos, como parecían hacer los realistas hasta ese momento, sería aceptable como un mecanismo simplificador y parsimonioso (Nye y Keohane, 1971a, pp. 343-45). Pero los hechos demostraban lo contrario: ya en esos años, era evidente que las tendencias en la política mundial socavaban esa visión con la emergencia de nuevos actores no estatales. Se decía, entonces, que la política mundial había cambiado, pero que los paradigmas conceptuales predominantes, como el pensamiento realista, no habían podido seguir el paso a esos cambios: "El clásico paradigma Estado-céntrico asume que los Estados son los únicos actores significativos en la política mundial..." (Nye y Keohane, 1971b, p. 721), algo inaceptable. La crítica continuó hacia finales de la década. En un libro publicado originalmente en 1977, sus autores propugnaban un nuevo tipo ideal de condiciones, opuestas a las teorías realistas contemporáneas, desde las cuales entender y explicar mejor la política internacional: la interdependencia compleja, un nuevo avance del descriptivismo teoricista que aspiraba a reflejar mejor, de manera más precisa, la importancia de las relaciones transnacionales y trasgubernamentales (véase Keohane y Nye 1988, cap. 2). A pesar de esto, el nuevo Realismo Estructural heredó y mantuvo el paradigma Estado-céntrico, fortaleciéndolo con un marco teórico más consistente. Si este enfoque ya no reflejaba lo que ocurría en el mundo, ¿por qué insistir en él?

El debate en torno a la utilidad y la pertinencia del paradigma Estado-céntrico reflejaba en realidad una discusión de fondo acerca de qué tanto debía una teoría ajustarse a la realidad. Autores como Robert Keohane y Joseph Nye (Poder $e$ interdependencia, 1988, capítulo 2) defendían la idea de que las teorías debían aspirar a describir exhaustivamente los hechos con el mismo nivel de complejidad que los

\footnotetext{
${ }^{10}$ Sobre la influencia del positivismo y sus variantes en Relaciones Internacionales, ver Smith (1996).
} 
caracterizaba en la realidad $^{11}$, una clara postura empirista que sería fuertemente criticada por Waltz en gran parte de su trabajo (véase p. ej. Waltz, 1997, p. 913 para una crítica explícita a ambos autores). Científicos y filósofos de la ciencia reconocen que los hechos no pueden comprenderse hasta no ser incluidos en un marco teórico. Según un reconocido manual de filosofía de la ciencia, "el conocimiento científico es el resultado de determinada práctica o actividad que podemos denominar, en sentido amplio, teorizar..." (Díez y Moulines, 1997, p. 17). De manera que defender la idea de que teoría y realidad son lo mismo, como ocurriría desde un sesgo descriptivista, supone defender la convicción de que a) el conocimiento puede obtenerse con la acumulación de información, llámense datos u observaciones, b) que éstos son independientes de todo conocimiento previo, y c) que por extensión las teorías, se construyen a partir de supuestas certezas que provienen de la experiencia. La conclusión inevitable de esta lógica es que, sorprendentemente, los hechos pueden llegar a comprenderse antes incluso de que se produzcan los mecanismos necesarios para su comprensión (Waltz, 1979, pp. 7/17-18). "Aquellos que creen extrañamente que el conocimiento empieza con certezas - dice Waltz- piensan en las teorías como edificios de verdades, los cuales pueden construir inductivamente" (Waltz, 1979, p. 5/14). Pero la comprensión de los hechos implica algo más que la suma de sus partes; comprender una clase de fenómenos supone algo más que la verificación, la constatación o la descripción, -incluso rigurosa, de sus componentes y asociaciones. Ya lo decía un prominente epistemólogo: “... explicar es responder a la pregunta ‘¿por qué?', es comprender y no sólo constatar” (Piaget, 1977, p. 11). Las teorías aspiran fundamentalmente a explicar algo, y para ello la sola descripción no es el mejor camino posible. "Una teoría, en lugar de ser un espejo a través del cual la realidad se refleja, es un instrumento a ser utilizado para explicar una porción de esa realidad, sobre cuyas verdaderas dimensiones nunca podremos estar seguros [...] Un instrumento de esa clase es de poca utilidad [explicativa] si hace poco más que imitar la complejidad del mundo" (Waltz, 1997, pp. 913-914). Posturas descriptivistas como la de Keohane y Nye, por ejemplo, de que a veces el paradigma de la interdependencia compleja se acerca más a la realidad que el realismo, y que contribuye así a supuestamente comprender mejor la política internacional, confunde los límites de lo que una teoría científica necesita describir y lo que no.

¿Es el paradigma Estado-céntrico una afirmación sobre cómo es el mundo realmente? Una interpretación descriptiva de las teorías en estricto conduce a entenderlas de modo cuasi literal. El paradigma Estado-céntrico, en cambio, es un supuesto pensado para captar mejor una de las características más importantes de la

${ }^{11}$ El libro Designing Social Inquiry (King et al., 1994) es otro trabajo frecuentemente citado por Waltz como ejemplo del empirismo predominante en la teoría de relaciones internacionales. En el libro, la observación y la experiencia están por delante de lo teórico: las teorías deberían ser tan complejas como lo sugieran las evidencias disponibles sobre lo que ocurre en el mundo empírico (véase King et al., 1994, p. 20), una posición que descansa sobre una concepción fundamentalmente descriptiva de la ciencia en la que teoría y realidad son equivalentes. Pero las teorías hacen lo contrario: seleccionan del mundo exterior, complejo e incierto, sólo aquellos elementos que se consideran cruciales para explicar ciertos fenómenos. Mearsheimer y Walt (2013, p. 431) recurren a una metáfora para ilustrar este punto: un mapa, p. ej., es útil en la medida en que cumple su propósito contando sólo con lo necesario -las preguntas serían entonces ¿cuál es ese propósito? y ¿qué es lo necesario?, pero es esto en donde precisamente las teorías se distinguen-. King y sus asociados, en cambio, asumen una posición que interpreta las teorías en términos descriptivos exhaustivos: si éstas presentan un modelo simple del mundo, dicen, en verdad estarían asumiendo que la propia realidad también es simple. La parsimonia se justifica, entonces, y según los autores, sólo cuando exista certeza -extrañamente a priori- de que la realidad es igualmente parsimoniosa. 
política internacional: que los Estados-nacionales son, efectivamente, los actores más importantes del sistema internacional. Las nociones teóricas son construcciones intelectuales, no se descubren desde la sola acumulación de datos. ${ }^{12} \mathrm{Se}$ trata de conceptos y supuestos que sirven específicamente a los propósitos del modelo que presenta cierta teoría en cuestión, y que responden con simpleza y elegancia a una realidad compleja y confusa. En ese sentido, decir que los Estados más importantes mantienen un rol central, aclara Waltz, no implica restar importancia o negar la existencia de otros actores no estatales (1979, p. 95/142). La visión Estado-céntrica no hace más que sugerir algo acerca de cómo la teoría representa la realidad. Eventualmente, Robert Keohane reconocería que la crítica de Waltz a su posición sobre el estatus del paradigma Estado-céntrico era "esencialmente correcta" (1993, p. 63, nota 9).

Otros autores críticos han mostrado una tendencia similar a interpretar el Realismo Estructural de manera literal. Sobre la "dependencia intelectual" de los países del sur, o periféricos, Arlene Tickner señala que "las categorías y los conceptos diseñados en el primer mundo pueden tener una escasa aplicación cuando estos se insertan en contextos sociales y culturales distintos, hasta tal punto que pueden volverse disfuncionales y contraproducentes". El predominio de la ciencia social occidental, y particularmente la ciencia social estadounidense, tendría el efecto de negar a los países de la periferia "la condición de sujetos activos en la construcción de su propio conocimiento" (Tickner, 2012, p. 163) ${ }^{13}$. No es de extrañar que la frase "sería absurdo construir una teoría de la política internacional basada en países como Malasia o Costa Rica” (Waltz, 1979, p. 72/109) sea una de las más criticadas en ese sentido. Pero, ¿hay en esto una voluntad de someter intelectualmente al llamado tercer mundo? Una lectura menos apasionada mostraría que lo que está detrás es un interés por comprender la política internacional como un sistema social. La frase de Waltz, de una crudeza ciertamente incómoda, sólo reconoce que en un mundo muy desigual unos influyen más

\footnotetext{
12 Que las nociones teóricas se construyan intelectualmente no significa que sean ideas arbitrarias, irreales, carentes de significado, o que hagan inconcebible toda coordinación con la experiencia. No se trata de renunciar a todo concepto que no correspondiera a aspectos 'observables', como señala el filósofo francés Gilles Gaston Granger. "Es bien evidente [...] que en la estructura de un conocimiento científico encontramos un gran número de conceptos que no tienen ningún examinando [sic] experimental directo, y que ni tan siquiera pueden tenerlo. Es que, en la medida en que la organización de una ciencia es la de un sistema simbólico [i.e., un modelo representativo], comporta necesariamente conceptos de tipo 'semántico', remitiendo más o menos directamente a aspectos de los fenómenos, y conceptos de tipo 'sintáctico', es decir, que juegan el papel de enlaces que combinan otros conceptos. Sólo un realismo [científico] furiosamente intemperante podría exigir que a estos conceptos sintácticos correspondiesen también aspectos del fenómeno o, peor aún, momentos absolutos de la realidad" (Granger, 1977, pp. 147, 154). La distinción que hace Waltz entre conceptos y supuestos como dos clases de nociones teóricas es coherente con esta oposición sintáctico-semántica de los conceptos teóricos que plantea Granger. "Una noción teórica puede ser un concepto, como la fuerza, o un supuesto, como el supuesto de que la masa se concentra en un punto". Y añade: los supuestos no son afirmaciones de hechos, “... son los elementos no-factuales de una teoría” (Waltz, 1979, pp. 5-6, 10/15, 22). Supuestos como el paradigma estado-céntrico, entonces, funcionan, dentro de los márgenes de la teoría, como conceptos más amplios que enlazan o combinan conceptos más específicos para crear elementos teóricos útiles. Los conceptos de tipo semántico, que en este esquema son las unidades menores, se construyen a la vez por abstracción, esto es, apelando por simplificación a las características o propiedades del fenómeno que resulten más relevantes al modelo en cuestión.

${ }^{13}$ Sin embargo, existen razones para dudar de que las categorías y conceptos producidos localmente, y particularmente en Sudamérica, escapen a ese mismo reproche.
} 
que otros sobre el contexto que los agrupa y los acontecimientos, un rasgo que ha sido recogido también por otras perspectivas.

Los actores internacionales más importantes, los Estados-nacionales, configuran a partir de sus interacciones el marco general para la vida internacional, algo que es cierto hoy y lo era también en 1979. Todos forman parte del conjunto, pero pocos tienen la capacidad para influir efectivamente en sus procesos y las tendencias generales dentro de él. Cada contexto, cada subregión, cada espacio de relaciones, presenta luego sus propios patrones y contingencias, pero la desigualdad es una cualidad inherente a la vida en sociedad: en política internacional, esas desigualdades se reflejan también en los contextos locales y regionales, no sólo a nivel global. El idealismo reivindicativo de Tickner es acrítico frente a esas desigualdades, y, sobre todo, frente a las expresiones de sometimiento político, económico e intelectual que provienen de las propias periferias. Así sucedía, por ejemplo, con la proyección exterior de Brasil en la región y la anterior creación de un "nuevo centro y nuevas periferias" como propósito no declarado, una estrategia que lleva una fuerte carga de nacionalismo y proteccionismo económico (sobre esto, véase Zibechi, 2013, caps. 6, 8 y 9). También es indiferente frente a lo que las diferencias de poder pueden llegar a incentivar entre los propios países periféricos. Recuérdese la intervención militar de Colombia en Ecuador en 2008, que ya presagiaba una actitud más asertiva y unilateral de ese país en cuanto a cuestiones de seguridad exterior. ¿Cómo ve el Realismo Estructural estas cuestiones? Reivindicaciones como esta suelen adquirir con facilidad un estatus convencional entre la academia local, quizás por razones emocionales o ideológicas. Si toda crítica es una contestación a los convencionalismos, el Realismo Estructural puede ayudar a asumir, de hecho, una actitud más crítica, algo imposible de reconocer si se le interpreta, equivocadamente, como una equivocada descripción del mundo.

\section{Explicación y modelos teóricos}

La interpretación literal de las teorías suele distorsionar lo que éstas establecen realmente sobre un conjunto de fenómenos de interés. Es un problema común no sólo en la evaluación de las teorías sino en su construcción formal y, sobre todo, en la manera como son presentadas. Por lo visto anteriormente, cabría aceptar que, si las teorías son en algún modo descriptivas, lo son sólo en la medida en que tratan de explicitar, aunque sin apelar a una descripción exhaustiva, lo que subyace a los hechos, renunciando a sus aspectos puramente circunstanciales y apelando a características generales con las cuales elaborar abstracciones. La descripción no es el principal propósito de las teorías; su principal propósito es la explicación. El conocimiento teórico en las ciencias empíricas busca explicar los hechos insertándolos en sistemas de ideas, lógicamente articulados, con cierto grado de generalidad. La explicación es el principal vínculo entre teoría y realidad. Esto abre una serie de interrogantes acerca de la capacidad explicativa de teorías altamente abstractas como el Realismo Estructural.

En primer lugar, existe una estrecha relación entre el tipo de descripción limitada que proporcionan los modelos teóricos, y la explicación. En una cita a Waltz en la sección anterior, se decía que una teoría "es una imagen, construida mentalmente, de cierto ámbito o dominio de actividad", y que, al enfatizar el rol de las imágenes y las representaciones en las teorías científicas, las bases epistemológicas del Realismo Estructural concuerdan con ciertos desarrollos en la filosofía de la ciencia según los cuales las teorías están constituidas básicamente por modelos, y que éstos son 
representaciones conceptuales del mundo empírico. El texto entonces prosigue de la siguiente manera:

Los infinitos materiales de cualquier ámbito de la realidad pueden ser organizados de muchas maneras diferentes. Una teoría indica que ciertos factores son más importantes que otros y especifica las relaciones que existen entre ellos. En la realidad todo está relacionado con todo lo demás, y un ámbito no puede ser separado de los otros. Lo que una teoría hace es aislar un determinado sector de todos los demás con el objetivo de ocuparse de él intelectualmente. Hacerlo es precondición para desarrollar una teoría que pueda explicar lo que ocurre dentro de él (Waltz, 1979, p. 8/19).

De esa manera, los modelos, las imágenes o las representaciones que caracterizan a una teoría hacen intelectualmente aprehensible el mundo real. Lo que parece desprenderse de esto es que la modelación de una teoría es necesaria para explicar los hechos. Pero estas ideas, contenidas en la cita anterior, son muy básicas y requieren por lo tanto ser ampliadas. La pregunta es ¿de qué forma un modelo teórico, siendo una construcción abstracta, puede llegar a explicar algo? La respuesta la da, en parte, la estructura de las teorías empíricas.

Los enfoques de la concepción semántica en filosofía de la ciencia, que enfatizan el rol de los modelos en el análisis de la naturaleza y la estructura de las teorías empíricas, no son una familia homogénea de teorías epistemológicas, pero sí comparten algunos elementos comunes. En primer lugar, presentar una teoría es presentar el conjunto de sus modelos característicos, y la determinación de esos modelos se hace mediante conceptos y principios o leyes (Díez, 1997, p. 51). En las concepciones semánticas, estos modelos teóricos, que son la conceptualización que proporciona una teoría sobre una determinada parcela del mundo empírico (Falguera, 1993, p. 174), constituyen el núcleo o parte formal de las teorías científicas. En segundo lugar, una vez sabido qué es y cómo funciona en abstracto una clase de fenómenos correspondientes a determinado ámbito de la realidad, lo siguiente en la identificación de una teoría consiste en la identificación de su parte aplicativa, llamada también modelo de datos o conjunto de aplicaciones intencionales. Este segundo elemento en la estructura de las teorías lo conforma el conjunto de fenómenos empíricos de los que se pretende dar cuenta aplicando los conceptos, principios y constricciones de la parte formal (Díez, 1997, p. 52; Guerrero, 2007, p. 28 y ss.) ${ }^{14}$. Ahora bien, en este enfoque explicar consiste en demostrar que entre un modelo teórico y un modelo de datos existe una cierta relación; que los fenómenos empíricos de la parte aplicativa pueden convertirse, con la

\footnotetext{
${ }^{14}$ Conviene hacer una precisión acerca de la naturaleza de los datos empíricos que utilizan las teorías. "Una aplicación no está dada independientemente de una conceptualización, sino que su descripción se da mediante un aparato conceptual. El dominio de aplicaciones intencionales no es 'ni realidad pura' ni 'pura experiencia', sino que una aplicación intencional se determina conceptualmente mediante el aparato conceptual de la teoría [el núcleo o modelo teórico] y mediante conceptos ya disponibles, los cuales son externos a la teoría en cuestión" y que ya existen en el edificio teórico existen en la disciplina (Guerrero, 2007, p. 34, comillas en el original). En otras palabras, "los datos [la base empírica de una teoría] están cargados de teoría, pero no de la teoría para la que son datos" (Díez y Moulines, 1997, p. 350; véase también Díez, 1997, p. 77). Este punto recoge el hecho de que en la ciencia empírica no existe tal cosa como la observación pura de los fenómenos, algo que se tuvo ya en cuenta en la formulación temprana del Realismo Estructural. "Científicos y filósofos de la ciencia -diría Waltz en un trabajo posterior- se refieren a los hechos como cargados de teoría, y a la teoría y los hechos como interdependientes" (Waltz, 1997, p. 913).
} 
ayuda de nociones teóricas, en modelos de la parte formal; o que tales fenómenos concretos satisfacen los conceptos y principios de la teoría y que se comportan de acuerdo con lo que en ella se establece (Cadevall, 1985, p. 15; Díez y Moulines, 1997, p. 332).

Desde este enfoque, la manera como teorías operan al explicar es identificar los objetos del mundo real y sus propiedades con los elementos del modelo teórico, sus conceptos y principios. Entonces, en vez de considerar directamente las relaciones empíricas que se dan entre los objetos reales estudiados, la atención del investigador se concentra, en cambio, en las relaciones que se dan entre los elementos teóricos que representan a esos objetos empíricos y sus propiedades, y con ello, indirectamente, se gana información acerca de los mismos objetos y sus propiedades representadas. Esta forma de utilizar los modelos teóricos para el estudio de la realidad es análoga a la manera como las ramas de la ciencia con un alto grado de matematización utilizan los modelos matemáticos para estudiar los hechos indirectamente a través de las relaciones y operaciones entre los números que representan a los objetos reales y sus propiedades (véase Díez y Moulines, 1997, pp. 121-122). Las teorías, entonces, se abstraen de la enorme complejidad de la realidad a través de imágenes (i.e. representaciones conceptuales) que la hacen intelectualmente más aprehensible.

Esta manera de entender la explicación desde una concepción semántica estructuralista de las teorías, que en cierta manera comparte el Realismo Estructural de Waltz, tiene dos importantes consecuencias para la evaluación teórica. Primero, descarta la vieja idea, muy arraigada en la práctica usual de la ciencia política, de que explicación y causalidad son lo mismo. Segundo, enfatiza la necesidad de evaluar las teorías en sus propios términos, considerando seriamente el conjunto de fenómenos para las que han sido formuladas realmente. Ambas cuestiones serán tratadas a continuación.

Explicación y causalidad no son sinónimos. En los programas de enseñanza suele decirse que el propósito de una investigación es descubrir relaciones del tipo A $\rightarrow$ $\mathrm{B}$, en la que el fenómeno o propiedad A es la causa del fenómeno o propiedad B. Pero en la realidad, y particularmente en la realidad social, sucede que los hechos tienen múltiples causas, por lo que la verdadera causa, o la causa total, de un hecho $\mathrm{B}$ sigue un esquema más cercano a este: $\mathrm{A}_{1}+\mathrm{A}_{2}+\mathrm{A}_{3} \ldots \mathrm{A}_{\mathrm{n}} \rightarrow \mathrm{B}$, donde lo que define el carácter causal de cada uno de esos factores es su carácter contrafáctico: si no hubiera ocurrido $\mathrm{A}_{\mathrm{n}}$, no habría ocurrido $\mathrm{B}$. La pregunta es ¿cómo decidir qué causa es la más importante? Tratar de entender algo apelando a la vez a todas las causas posibles es una tarea tediosa e inútil porque no genera un conocimiento real sobre el porqué de las cosas. La causalidad por sí sola no resuelve el problema de explicar los hechos. La explicación se vale de esas causas, pero no de todas; pone de manifiesto una puesta en orden entre los factores determinantes de un modelo: "la explicación causal de un suceso no tiene por qué referirse a todas sus causas, sino por lo general a aquella o aquellas más destacadas en el particular contexto explicativo" (Díez y Moulines, 1997, p. 154). El resultado es la "diversidad de la tarea investigadora" en las ramas de la ciencia que se ocupan de sucesos causalmente complejos, algo que ha sido comparado con una suerte de división social del trabajo: la existencia de diversas teorías, cada una de las cuales trata sobre uno o más factores causales; cada una elaborando explicaciones apelando a causas distintas, que no tienen por qué ser incompatibles porque cada una es válida en su contexto, y en conjunto, pueden hasta resultar complementarias (ibíd., p. 157). Respecto a esto, entonces, la explicación consistiría, en parte, en establecer una 
jerarquía entre las diversas causas de un hecho. Esta es una situación común en la ciencia política, y en particular en la teoría política internacional, donde la competencia entre distintas propuestas teóricas suele ser intensa.

Ahora bien, ¿por qué sería relevante para el Realismo Estructural en Relaciones Internacionales dedicar tiempo a reflexionar sobre estas cuestiones? Porque una de las más básicas objeciones que se han planteado a la teoría, y en general a otras teorías sistémicas en la disciplina, es que las variables de tipo sistémico o estructural raramente determinan la naturaleza de la política internacional, y aún menos la política exterior de los Estados (véase Jervis, 1997, p. 103 y ss.). Este tipo de críticas siguen el viejo esquema $\mathrm{A} \rightarrow \mathrm{B}$, desde el cual se espera que las causas de un hecho no sólo sean necesarias sino también suficientes, y que sean en ese sentido determinantes. Llevadas al extremo, son una interpretación casi literal de las teorías como esquemas descriptivos exhaustivos que establecen relaciones directas entre dos o más fenómenos. Sin embargo, aunque toda causa es de alguna manera necesaria, ninguna es suficiente por sí sola. "A través de una teoría se pone de manifiesto el significado de lo observado...", decía Waltz; las teorías ordenan los hechos particulares de un cierto ámbito de manera que puedan ser vistos como mutuamente dependientes; conectando hechos que de otra forma resultarían dispares e inconexos (1979, pp. 9-10/21; 1988, p. 615). Si se espera que una teoría sistémica explique los fenómenos estableciendo sólo relaciones causales directas, perdurará la sensación de que la teoría tiene poco que decir sobre algo en concreto.

\section{Los alcances de la explicación y la naturaleza dual de los fenómenos políticos}

Si el Realismo Estructural no sigue el esquema de causalidades directas, como tampoco lo hacen otras teorías científicas, ¿qué explica y cómo lo hace? Para responder a esta pregunta, recuérdese lo visto en la sección anterior. Lo dicho acerca de la concepción semántica de las teorías en la filosofía de la ciencia ha puesto énfasis en los aportes del estructuralismo epistemológico. En la concepción estructuralista, una teoría, también llamada elemento teórico, está constituida en un sentido mínimo por una parte formal, o núcleo, y una parte aplicativa, o dominio de aplicaciones intencionales. La parte formal contiene tanto los elementos que permiten caracterizar el ámbito de estudios, o las partes de la realidad que contienen las diversas aplicaciones de la teoría, como los principios y leyes que rigen en él. La parte aplicativa contiene el conjunto de fenómenos empíricos a los cuales se pretende aplicar los elementos de la parte formal. Una teoría se identifica entonces en su forma más simple por el par formado por un núcleo (K) y un dominio de aplicaciones (I): T $=\langle\mathrm{K}$, I $\rangle$ (Díez y Moulines, 1997, p. 375). Este esquema básico sobre la estructura formal de las teorías es suficientemente útil para entender que el conjunto de fenómenos sobre los que una teoría trata están determinados por su propio aparato conceptual. De manera que al identificar lo que una teoría intenta explicar en primer lugar, es importante prestar atención a la manera como conceptualiza los fenómenos de su interés. En el caso del Realismo Estructural, los fenómenos políticos internacionales son conceptualizados como entidades esencialmente duales ${ }^{15}$.

\footnotetext{
${ }^{15}$ Las teorías deben evaluarse en sus propios términos. Antes, deben identificarse los rasgos que definen su propósito y sus alcances y limitaciones. Pero la mayoría de las veces esto no ocurre. En los estudios políticos internacionales, por ejemplo, la clasificación de las teorías según grandes debates interteóricos -una forma común de enseñanza y aprendizaje que predomina en los manuales y programas de
} 
El Realismo Estructural es una aproximación a la dimensión sistémica de los fenómenos internacionales, a la manera como las características del sistema político internacional, a través del análisis de su estructura, inciden sobre ellos y cómo el propio desarrollo de esos fenómenos incide sobre la configuración del propio sistema político (en un proceso de recursividad sistémica). Para comprender esta relación es preciso aislarse de las circunstancias, las particularidades y los elementos puramente accidentales y encarar los fenómenos en sí mismos, es decir, como hechos genéricos. Esta manera de proceder no es extraña en las ciencias sociales. El estructuralismo social se originó con los trabajos de Claude Lervi-Strauss y la antropología estructural. Pero el mismo Waltz reconocía que su principal fuente de inspiración la obtuvo de la lingüística estructural de Saussure, cuyo enfoque buscó incorporar luego la antropología cultural (Gómez García, 1981, pp. 33-34) ${ }^{16}$. Así pues, el Realismo Estructural de Waltz, como otras formas de estructuralismo en las ciencias sociales, están presentes reminiscencias y herencias lingüísticas y antropológicas.

En primer lugar, los hechos de política internacional son, en lo esencial, hechos sociales. En la sociología clásica, un hecho social por definición mantiene un carácter general dentro de la extensión de una sociedad y una existencia propia (objetiva), independiente de sus manifestaciones individuales (Durkheim, [1895] 1978, p. 42). La lingüística clásica, por otra parte, elabora una distinción similar al separar lengua y habla: la lengua es el producto social de la facultad del lenguaje, que es individual; es un conjunto de convenciones adoptadas por el cuerpo social para permitir el ejercicio de esa facultad en los individuos. De esa forma, "al separar la lengua del habla, se separa a la vez: $1^{\circ}$ lo que es social de lo que es individual; $2^{\circ}$ lo que es esencial de lo que es accesorio y más o menos accidental” (Saussure, 1945, pp. 39, 41).

En el Realismo Estructural de Waltz, los fenómenos políticos internacionales son concebidos, primero, como hechos sociales, que son por definición genéricos. Luego son definidos como hechos particulares, que responden a las circunstancias inmediatas y situaciones concretas. Esta oposición de concepciones es lo que en un trabajo anterior se ha llamado la naturaleza dual de los fenómenos políticos

estudio- sólo contribuye a reforzar los estereotipos que las reducen a esquemas interpretativos muy simples, pero manejables desde un punto de vista puramente instrumental. El resultado: su naturaleza se reduce a una interpretación casi descriptiva de la realidad, sus propósitos se distorsionan, y se desconocen cuáles son sus alcances y limitaciones. Jack Snyder (2004) proporciona un ejemplo de esto en One World, Rival Theories, un breve artículo ampliamente difundido. En esta visión de las cosas, la adhesión a una teoría en particular termina por asemejarse más a una cuestión de fe. Frente a este problema, la pregunta básica para guiar la evaluación de las teorías sigue siendo más bien simple e intuitiva: ¿qué es lo que dicen y qué es lo que no dicen las teorías sobre los acontecimientos?

${ }^{16}$ Un correcto entendimiento del estructuralismo en Relaciones Internacionales, como en otras ciencias sociales básicas o derivadas, no estaría completo sin remitirse a las raíces heredadas de las perspectivas lingüísticas, particularmente la fonología de N. Trubetzkoy. A decir del propio Levi-Strauss, el método de la nueva fonología se basaba en cuatro pasos fundamentales: "pasa del estudio de los fenómenos lingüísticos 'conscientes' al de su estructura 'inconsciente'; rehúsa tratar los 'términos' como entidades independientes; y toma como base de su análisis, por el contrario, las 'relaciones' entre los términos; introduce [finalmente] la noción de "sistema" (Levi-Strauss, 1995, p. 76). La fonología de Trubetzkoy, antecedente de los modernos análisis estructurales de los fenómenos sociales, no se limitaba a declarar que los fonemas, como los agentes o los hechos sociales, son siempre parte de un sistema, sino que mostraba sistemas concretos poniendo en evidencia su estructura a partir de relaciones explícitas entre esos elementos. Con este nuevo método de análisis, las ciencias sociales lograban formular relaciones necesarias. El análisis estructural de Waltz, y la teoría derivada de sus abstracciones, es heredero de este enfoque. 
internacionales (véase Morales Giraldo, 2014), y se encuentra en el núcleo (K) de la teoría de Waltz. Su situación, entonces, la convierte en una noción central en la conceptualización del tipo de fenómenos (I) de los que la teoría trata de dar cuenta. La teoría no trata, por ejemplo, sobre cuestiones como la guerra entre dos o más países en determinado lugar y momento, sino de la guerra como fenómeno socio-político en el sistema internacional, de su explicación a través de casusas permisivas -que la convierten en una posibilidad siempre latente- y no de sus causas más inmediatas (Waltz, 1959, p. 232). Esto implica una enorme diferencia en la manera de ver las cosas: como fenómeno particular, la guerra puede resultar ser infrecuente, improbable y excepcional; como fenómeno estructural, en cambio, resulta ser un patrón recurrente y hasta habitual. En el primer caso interesaría explicar la guerra, en el segundo, la paz. Esta distinción, que es la esencia de la naturaleza dual de los fenómenos políticos internacionales, se encuentra en el núcleo del estructuralismo de Waltz, definiendo su propósito y señalando sus alcances y limitaciones.

En The Origins of War in Neorealist Theory (Waltz, 1988), por ejemplo, la paz entre las grandes potencias durante los años de la Guerra Fría es explicada tanto desde un punto de vista sistémico -según como lidian con la inestabilidad y la competencia en un sistema bipolar o en uno multipolar- como desde el punto de vista de las particularidades de los propios Estados y sus relaciones — según como ciertas tecnologías y estrategias, como las armas y la disuasión nucleares, elevan los costos de la violencia-. En The Myth of National Interdependence (Waltz, [1970] 2008), la importancia política, y no sólo económica, de la interdependencia se hace evidente al indagar por las implicancias de las relaciones entre comercio internacional e inversión extranjera entre países con una enorme disparidad de poder. Al introducir el carácter asimétrico inherente al conjunto de Estados en la visión que se tiene de la interdependencia económica, el panorama resulta distinto: en lugar de una red de relaciones económicas interdependientes surge un escenario dispar en el que unos son más dependientes o independientes que otros. En Conflict in World Politics (Waltz, [1971] 2008c) la explicación del conflicto asume una perspectiva estructural que busca encontrar una razón a este tipo de situaciones más allá de las circunstancias que envuelven conflictos particulares. Finalmente, la teoría del balance de poder, dentro del programa de investigación neorrealista, es también una aproximación a la dimensión estructural de este tipo de sucesos, no a sus particularidades: "la teoría del balance de poder explica un resultado -la formación recurrente de balances de poder-, que puede no coincidir con las intenciones de cualquiera de las partes cuyas acciones se combinan para producir ese resultado" (Waltz, 1979, p. 119/175-176). Cada uno de estos tipos de fenómenos: la paz, la interdependencia, el conflicto y el balance de poder, son estudiados no como hechos particulares sino como procesos sistémicos.

Una última observación sobre la manera como los componentes formales de una teoría determinan el alcance de la explicación. Un mal entendimiento sobre qué es lo que las teorías explican realmente puede generar simplificaciones. El Neorrealismo en particular ha sido objeto de una clara malinterpretación: la idea, ampliamente difundida, de que se trata de una teoría orientada a temas militares y de seguridad. Uno de los textos introductorios más leídos en los cursos de relaciones internacionales en los países de habla hispana, por ejemplo, reproduce esta lectura errada: entre las teorías realistas, señala el manual, la problemática de estudio se centra en la seguridad nacional (cf. 
Barbé 1995, 60-64) ${ }^{17}$. Es cierto que en el programa teórico neorrealista se asume que la supervivencia, y por lo tanto la seguridad, es uno de los intereses fundamentales de los Estados (Waltz, 1979, pp. 91-92). Sin embargo, esta no es una afirmación empírica sino un supuesto teórico, una abstracción. Si la teoría restringiera la comprensión de los fenómenos a aspectos de seguridad de tipo militar, su visión del mundo sería la de un reduccionismo poco convincente. Pero el carácter progresivo que la teoría ha demostrado desde su aparición indica lo contrario. Decir que el Neorrealismo es una teoría que tiene a los asuntos militares como problemática central equivale a negar las contribuciones que se han hecho desde este enfoque, por ejemplo, al estudio de la política tras la interdependencia económica (véase Waltz, [1970] 2008a; 1979, pp. 139146/206-215; 2000, pp. 14-18; Baldwin, 1980, p. 486 y ss.), del problema de la distribución relativa de las ganancias bajo esquemas de cooperación en ausencia de un gobierno común (véase Grieco, 1988a; 1988b; y Grieco, Powell y Snidal 1993), o al estudio crítico y normativo de la política exterior norteamericana, con especial atención a los riesgos del unilateralismo (véase Waltz 2000, pp. 27-39; 2004).

\section{6. ¿Qué predicen las teorías científicas?}

El conocimiento científico tiene como otra de sus principales características la predicción (Bunge, 1997, p. 41). Pero, así como se espera que las teorías se refieran a hechos particulares, en la descripción o en la explicación, también se espera que elaboren predicciones precisas sobre acontecimientos específicos. Sin embargo, “... la predicción científica, en contraste con el tipo de predicción que proviene de las profecías [...] no es del tipo 'ocurrirá e' sino más bien de este otro: 'ocurrirá e (de la clase E) siempre que suceda c (de la clase C), pues todo hecho de clase C es acompañado o seguido por un hecho de clase E" (Bunge, 1997, p. 42). Esto último sugiere dos cosas importantes acerca de la predicción en las ciencias empíricas: la generalidad de las prospecciones, y su relación estrecha con la explicación. El siguiente pasaje recoge algunas ideas importantes sobre este asunto:

“... incluso en las ciencias de la naturaleza, las predicciones que cabría esperar en justicia no conducen sino excepcionalmente a acontecimientos en sentido estricto. El físico, el químico, el biólogo, se contentan por lo general con prever tipos de acontecimientos y tal es, creemos, lo que se puede esperar razonablemente de la ciencia. Es cierto que en estos últimos casos la determinación de los tipos de acontecimientos es a menudo tan rigurosa, a escala

\footnotetext{
${ }^{17}$ Incluso dentro de la tradición de pensamiento realista, que incluye a autores anteriores a la Guerra Fría, es difícil sostener -si no imposible- que la seguridad nacional sea el centro de interés. En La crisis de los veinte años (1919-1939) ([1939] 2004), texto clásico de E. H. Carr, publicado originalmente en 1939, un concepto tan frecuentemente asociado al realismo como el de poder es definido no sólo en función del poder militar sino también en función del poder económico y en función al poder sobre la opinión y las ideas (véase, Carr [1939] 2004, cap. 8). Lo mismo ocurre con Hans Morgenthau, en cuyo clásico La política entre las naciones ([1948] 1986), los conceptos de poder político y poder militar no son de ninguna forma equivalentes: "El poder político debe distinguirse de la fuerza [...] Cuando la violencia se convierte en realidad, el poder político abdica a favor del poder militar...", sin embargo, añade el autor, "especialmente en política internacional la fuerza armada como amenaza real o potencial es el componente material más importante que contribuye a conformar el poder político de una nación" (Morgenthau [1948] 1986, p. 43). Si la política internacional, como toda política, es una lucha por el poder, tal como la define el autor, entonces se trata en realidad de una lucha por el poder político. La esencia de la política internacional entre los autores realistas de todos los tiempos y tendencias ha sido siempre política y no militar.
} 
de nuestras observaciones, nuestro poder de disociación de sus circunstancias tan amplio, que la predicción equivale prácticamente a la de los acontecimientos singulares. No ocurre lo mismo es las ciencias sociales..." (Granger, 1977, p. 149, énfasis en el original).

Las teorías científicas predicen tipos de acontecimientos, no acontecimientos particulares, porque las teorías mismas tratan con abstracciones con un grado mayor de generalidad respecto a acontecimientos particulares. Esto resulta mucho más claro al reconocer la relación que existe entre predicción y explicación. De nuevo, resulta útil referirse a ciencias en las que estas cuestiones están más y mejor desarrolladas.

En la antropología estructural, por ejemplo, "el análisis de un sistema o el de los elementos de un grupo de mitos, seguramente no conduce a establecer una previsión que enuncie 'lo que va a pasar' en un momento ulterior. No obstante, en el caso en que este análisis pueda ser llevado a término, construye un sistema -una teoría- más o menos cerrado de posibilidades...". Esto es análogo a lo que ocurrió en la construcción y posterior aprovechamiento de la tabla periódica de los elementos químicos de Mendeleieff: "Un conjunto de fenómenos -elementos químicos- conocidos y suficientemente analizados es estructurado según determinados conceptos; esta estructuración hace aparecer objetos abstractos - nuevos elementos químicos- que la experiencia no ha desvelado, pero para los que lo cerrado del sistema -en este caso, la teoría- exige que existan fenómenos en consonancia" (Granger, 1977, p. 150). Las teorías, entonces, por medio de la explicación, conforman esquemas cerrados de posibilidades, lo que permite la anticipación de cierto tipo de acontecimientos excluyendo la posibilidad de otros. La explicación de hechos particulares y la predicción comparten la misma estructura lógica ${ }^{18}$. Una vez que un fenómeno particular llega a comprenderse, una vez que se han dilucidado sus mecanismos subyacentes ${ }^{19}$, los eventos particulares que tienen lugar en ese campo de la realidad, se vuelven previsibles. Las teorías, por medio de la explicación, elaboran asociaciones entre los factores más relevantes de un ámbito del mundo empírico, establecen jerarquías entre esas variables o causas y brindan una imagen de cómo es que funciona la realidad. La predicción se obtiene como un producto del conocimiento obtenido sobre las regularidades que dan sentido a nuestra comprensión de las cosas. Este tipo de predicción, vale aclarar, no depende del tiempo: no se espera que los eventos previstos ocurran en cierto momento, sino bajo ciertas circunstancias.

El Realismo Estructural explica, por ejemplo, por qué el balance de poder constituye un proceso sociopolítico recurrente en la historia, y es esta explicación la que permite construir un marco de condiciones generales que permiten, a la vez, inferir la formación de un nuevo balance de poder en el futuro. La teoría es incapaz de decir cuándo es que tendrá lugar o qué es lo que cada país en particular hará al respecto, sólo se limita a anticipar un tipo de situación o suceso, que en este caso es un patrón de

\footnotetext{
18 Según la tesis de Hempel sobre la simetría entre explicación y predicción, "la explicación de fenómenos empíricos particulares y la predicción tienen la misma estructura lógica, la única diferencia entre ambas es pragmática y tiene que ver con la relación temporal entre la ocurrencia del hecho particular y la construcción del argumento [...] Las predicciones son pues retrodicciones, 'predicciones' de hechos conocidos; las predicciones, si llegan a confirmarse, son explicaciones 'avanzadas"” (Diez y Moulines, 1997, p. 239).

19 Sobre la explicación por mecanismos, y sobre el concepto mismo de "mecanismos" como procesos concretos en sistemas reales, véase Bunge (1997b, pp. 5-10, 28-35, 37-45).
} 
interacción entre las potencias más importantes del sistema internacional, o de los subsistemas regionales, una tendencia a iniciar políticas de balance o contención bajo ciertas condiciones (Waltz, 2000, pp. 30, 38). No hace falta decir que las dinámicas políticas más recientes a nivel global y regional parecen confirmar las previsiones neorrealistas sobre estas regularidades. Cabría entender, finalmente, que la estructura lógica de las predicciones implica para el investigador un conjunto de posibilidades hipotéticas de las cuales pueden inferirse situaciones reales bajo ciertas condiciones.

\section{Predicciones y creencias: el Realismo Estructural y el final de la Guerra Fría}

El primer capítulo de Theory of International Politics no dice mucho acerca del carácter predictivo de las teorías. Sin embargo, irónicamente una de las mayores críticas a la teoría tiene que ver con la anticipación de un gran suceso que sus proponentes no pudieron prever: el final de la Guerra Fría. Tras el rápido declive y posterior desaparición de la Unión Soviética, en los estudios internacionales las críticas se orientaron principalmente a señalar la incapacidad de las teorías de relaciones internacionales, y en espacial de las teorías realistas, para anticipar el final de la Guerra Fría. "Los cientistas políticos y sus teorías -se dice- fracasaron no sólo en anticipar cualquiera de los dramáticos eventos de los últimos años sino también en reconocer la posibilidad de que esos cambios tuvieran lugar" (Lebow y Risse-Kappen, 1995, p. 1). ¿Por qué las teorías de política internacional no pudieron prever un cambio tan drástico? La cita anterior contiene dos elementos cuyo análisis puede ayudar a responder en parte esa pregunta: a) la capacidad predictiva de las propias teorías, que ya ha sido descrita brevemente en la sección anterior, y b) la disposición por parte de quienes hacen uso de esas teorías para reconocer la posibilidad de ciertos eventos. En este caso en particular lo que ocurrió fue que lo segundo terminó por condicionar la efectividad de lo primero.

Muchas veces ciertos hechos parecen ser imposibles o tan improbables que no son tomados en cuenta seriamente, incluso cuando las teorías dejan abiertas esas posibilidades, y éste es uno de los principales aspectos a tener en cuenta al evaluar la capacidad predictiva de una teoría: las ideas preconcebidas pueden obstaculizar la capacidad de predicción. En The Stability of a Bipolar World ([1964] 2008b), por ejemplo, el propio Waltz decía que no había ninguna duda acerca de la durabilidad del bipolarismo a largo plazo dada las condiciones existentes hasta ese momento. Quince años después, en 1979, la situación parecía seguir siendo la misma a los ojos del autor (véase Waltz, 1979, p. 176-183/259-268). Sin indicios de ningún tipo, los realistas no mostraron un interés real en plantearse la posibilidad del colapso de la Unión Soviética y el final de la Guerra Fría. Pero, ¿qué decía la teoría al respecto? En un mundo bipolar, el colapso de una de las dos potencias que sostienen ese orden supondría un cambio a nivel sistémico o estructural. El Realismo Estructural explica ese tipo de cambios a partir del cambio en uno de los principales elementos estructurales del sistema internacional: la distribución de capacidades, que es, además, su elemento más dinámico (véase Waltz, 1979, p. 97, 101/145, 150). La Guerra Fría, decía Waltz en una referencia explícita a este caso, “... está firmemente arraigada en la estructura de la política internacional de la posguerra, y durará tanto como perdure esa estructura" (Waltz, 1988, p. 628). La Guerra Fría culminaría, entonces, cuando la estructura bipolar que la sostenía comenzara a erosionarse, como ocurrió con el rápido declive y la posterior desaparición de la Unión Soviética, lo que generó una rápida reconfiguración en la distribución de capacidades a nivel global. De manera que el cambio sistémico era un tipo de situación ya prevista por la teoría, y aun presente en sus formulaciones más 
tempranas. Era una de sus aplicaciones posibles, pero hasta finales de la década de 1980 permaneció como un potencial no explorado. Las predicciones se limitaban a prever un tipo de situación hipotética, pero ningún teórico realista asumió seriamente la necesidad de desarrollar una teoría específica o hipótesis auxiliar como parte del programa investigación neorrealista que permitiera anticipar con algo más de detalle los cambios a nivel estructural. Sin indicios razonables para dudar de lo contrario, la confianza en la estabilidad y la durabilidad del orden bipolar se reforzó, y esta creencia se mantuvo firme hasta el momento en que sucedió el cambio. Fueron las convicciones de los investigadores y teóricos las limitaron el potencial predictivo de la teoría.

\section{Reflexiones finales}

Theory of International Politics nos presenta una introducción a los rasgos generales de las teorías científicas y su papel fundamental en la ciencia moderna. En ese aspecto, cumple un propósito más bien didáctico: anima al lector a reflexionar sobre los aspectos epistemológicos de la teoría política. Los textos citados a lo largo de este trabajo contribuyen a ampliar estas ideas de manera somera. Quizás la lección más importante que puede extraerse de la obra de Watlz es que las teorías, aun antes de que la propia investigación cobre forma, cumplen la función de dibujar el horizonte del investigador, guiando la observación y señalando las problemáticas de interés. Tanto los científicos como los filósofos de la ciencia saben esto. El rol de las teorías no empieza ni termina en el análisis o en la comprensión de los hechos: comienza mucho antes, modelando las intuiciones, sirviendo de base para identificar esos mismos hechos (Chalmers, 2000, cap. 1), y redirigiendo el interés de toda una comunidad científica hacia nuevas problemáticas de las que antes no se tenía conciencia.

Finalmente, llevando estas reflexiones a terrenos más cercanos a nuestra realidad, los temas discutidos en este trabajo, a consideración de la obra de Kenneth Waltz, pueden abrir espacios de discusión sobre la situación actual y el futuro de las Relaciones Internacionales en Sudamérica. En la región, la labor de la política internacional ha estado orientada en los últimos años al análisis casi exclusivo de las coyunturas. Y ese interés por las coyunturas, en parte, explica la escasa producción teórica en la región, muy aparte de las viejas reivindicaciones frente al colonialismo intelectual y el dominio sobre las ideas. Pero para comprender los fenómenos que afectan nuestro entorno directamente, y adquirir así cierta certeza acerca de su desarrollo futuro, los análisis de coyuntura son insuficientes. Hace falta teoría. Al no integrar el conocimiento inmediato sobre acontecimientos en marcha dentro de marcos teóricos más generales, los cientistas políticos han visto con cierto desconcierto, por ejemplo, cómo la integración en la región ha fracasado y los liderazgos emergentes se han replegado. Es en estos momentos que proliferan títulos como el fracaso de la integración, integración: ¿mito o realidad?, o la falta de liderazgo en..., todos respondiendo a una coyuntura específica. Teorías de diverso origen, en cambio, podrían haber previsto estos sucesos, no con exactitud, sino estableciendo un conjunto de posibilidades desde las cuales inferir escenarios sobre los plantear espacios de trabajo y debate. Los alcances filosóficos del Realismo Estructural invitan a asumir posturas críticas respecto a un conocimiento que damos por sentado. 


\section{Referencias}

Ashley, R. K. (1986). The Poverty of Neorealism. En R. O. Keohane (Ed.), Neorealism and its Critics (pp. 255-300). Nueva York: Columbia University Press.

Baldwin, D. A. (1980). Interdependence and Power: A Conceptual Analysis. International Organization, 34(4), 471-506.

Barbé, E. (1995). Relaciones Internacionales. Madrid: Tecnos.

Bunge, M. (1972). Teoría y realidad. Barcelona: Ariel.

Bunge, M. (1982). Epistemología. La Habana: Editorial de Ciencias Sociales.

Bunge, M. (1997a). La ciencia. Su método y su filosofía (2da edición). Buenos Aires: Editorial Sudamericana.

Bunge, M. (1997b). Mechanism and Explanation, Philosophy of the Social Sciences, 27 (4), 410-465.

Bunge, M. (1999). Las ciencias sociales en discusión. Una perspectiva filosófica. Buenos Aires: Editorial Sudamericana.

Buzan, B. (1996). The Timeless Wisdom of Realism? En Steve Smith, Ken Booth y Marysia Zalewski (Eds.), International theory: positivism and beyond (pp. 47-65). Cambridge: Cambridge University Press.

Cadevall, M. (1985). El concepto de explicación en la concepción estructuralista. Enrahonar: quaderns de filosofía, (12), 11-18.

Carr, E. H. [1939] (2004). La crisis de los veinte años (1919-1939). Una introducción al estudio de las Relaciones Internacionales. Madrid: Catarata.

Chalmers, A. (1984). ¿Qué es esa cosa llamada ciencia? Una valoración de la naturaleza y el estatuto de la ciencia y sus métodos. Madrid: Siglo XXI Editores.

Del Arenal, C. (2007). Introducción a las relaciones internacionales. Madrid: Tecnos.

Díez, J. A. (1997). La concepción semántica de las teorías científicas. Éndoxa: Series Filosóficas, (18), 41-91.

Díez, J A., y C. U. Moulines (1997). Fundamentos de filosofía de la ciencia. Barcelona: Editorial Ariel, S.A.

Durkheim, É. [1895] (1978). Las reglas del método sociológico. Madrid: Ediciones Morata.

Elman, C. y M. Elman (2003). Introduction: Appraising Progress in International Relations Theory. En C. Elman y M. Elman (Eds.), Progress in International Relations Theory: Appraising the Field (pp. 1-20). Cambridge: MIT Press.

Falguera, J. L. (1992). La noción de modelo en los análisis de la concepción estructuralista. Ágora, 11(1), 97-104. 
Falguera, J. L. (1993). El enredo de los modelos en los análisis de las ciencias factuales. Ágora, 12(2), 171-178.

Falguera, J. L. (1994). Unidad de noción bajo los usos del término 'modelo' en las ciencias matemáticas y factuales. Contextos, XII(23-24), 221-244.

Gilpin, R. G. (1986). The Richness of the Tradition of Political Realism. En R. O. Keohane (Ed.), Neorealism and its Critics (pp. 301-321). Nueva York: Columbia University Press

Gómez García, P. (1981). La antropología estructural de Claude Levi-Strauss. Ciencia, filosofía, ideología. Madrid. Editorial Tecnos.

Granger, G. G. (1977). La explicación en las ciencias sociales. En J. Piaget (Ed.), La explicación en las ciencias (pp. 143-160). Barcelona: Ediciones Martínez Roca S.A.

Grieco, J. M. (1988a). Anarchy and the Limits of Cooperation: A Realist Critique of the Newest Liberal Institutionalism. International Organization, 2(3), 485-507.

Grieco, J. M. (1988b). Realist Theory and the Problem of International Cooperation: Analysis with an Amended Prisoner's Dilemma Model. The Journal of Politics, 50(3), 600-624.

Grieco, J. M., Powell, R. y Snidal D. (1993). The Relative-Gains Problem for International Cooperation. The American Political Science Review, 87(3), 727-43.

Guerrero, G. (2007). "Van Fraassen y la concepción estructuralista de las teorías". Praxis Filosófica, (25), 21-38.

Hawking, S. W. (1999). La teoría del todo. El origen y el destino del universo. Barcelona: Debolsillo.

Jervis, R. (1997). System Effects. Complexity in Political and Social Life. Princeton, New Jersey: Princeton University Press.

Jervis, R. (2003). Realism, Neoliberalism and Cooperation: Understanding the Debate. En C. Elman y M. Elman (Eds.), Progress in International Relations Theory: Appraising the Field (pp. 277-309). Cambridge: MIT Press.

Keohane, R. O., y Nye, J. S. [1977] (1988). Poder e Interdependencia: La Política Mundial en Transición. Buenos Aires: Grupo Editor Latinoamericano.

Keohane, R. O. (1993). Instituciones internacionales y poder estatal: Ensayos sobre teoría de las Relaciones Internacionales. Buenos Aires: Grupo Editor Latinoamericano.

King, G., Keohane, R. O. y Verba, S. (1994). Designing Social Inquiry: Scientific Inference in Qualitative Research. Princeton: Princeton University Press.

Lakatos, I. (1989). La metodología de los programas de investigación científica. Madrid: Alienza Editorial.

Lebow, R. N. y Risse-Kappen, T. (1995). Introduction: International Relations Theory and the End of the Cold War. En R. Ned Lebow y T. Risse-Kappen (Eds.), International 
Relations Theory and the End of the Cold War (pp. 1-21). New York: Columbia University Press.

Levi-Strauss, C. (1995). Antropología estructural. Barcelona: Paidós.

Lobell, S. E. (2010). Structural Realism/Offensive and Defensive Realism. En R. A. Denemark (Ed.), The International Studies Encyclopedia (pp. 6651-6669). Volumen X. Chichester: Wiley-Blackwell.

Mearsheimer, J. J., y S. Walt. (2013). Leaving theory behind: Why simplistic hypothesis testing is bad for International Relations. European Journal of International Relations, 19(3), 427-457.

Morales Giraldo, J. F. (2014). La competencia en seguridad y la comunidad sudamericana: teoría y crítica. Revista Andina de Estudios Políticos, IV (2), 73-98.

Morgenthau, H. [1948] (1986). Política entre las Naciones. La lucha por el poder y la paz. Buenos Aires: Grupo Editor Latinoamericano.

Nye, J. S., y Keohane, R. O. (1971a). Transnational Relations and World Politics: An Introduction. International Organization, 25(3), 329-349.

Nye, J. S., y Keohane, R. O. (1971b). Transnational Relations and World Politics: A Conclusion. International Organization, 25(3), 721-748.

Piaget, J. (1977). Introducción: el problema de la explicación. En J. Piaget (Ed.), La explicación en las ciencias (pp. 11-21). Barcelona: Ediciones Martínez Roca S.A.

Saussure, F. de (1945). Curso de lingüística general. Traducción de Amado Alonso. Buenos Aires: Editorial Losada S.A.

Smith, S. (1996). Positivsm and beyond. En Steve Smith, Ken Booth y Marysia Zalewski (Eds.), International theory: positivism and beyond (pp. 11-44). Cambridge: Cambridge University Press.

Snyder, J. (2004). One World, Rival Theories. Foreign Policy, (145), 52-62.

Tickner, A. B. (2012). Relaciones de conocimiento centro-periferia: hegemonía, contribuciones locales e hibridización. Politai: Revista de Ciencia Política, 3(4), 163172.

Vasquez, J. A. (1997). The Realist Paradigm and Degenerative versus Progressive Research Programs: An Appraisal of Neotraditional Research on Waltz's Balancing Proposition, The American Political Science Review, 91(4), 899-912.

Viotti, P. R., y Kauppi, M. V. (2012). International Relations Theory. Boston: Longman.

Wæver, O. (2009). Waltz's Theory of Theory, International Relations, 23(2), 201-222.

Walt, S. (1997). The Progressive Power of Realism, The American Political Science Review, 91(4), 931-935.

Waltz, K. N. (1959). Man, the State and War. New York: Columbia University Press. 
Waltz, K. N. [1964] (2008b). The Stability of a Bipolar World. En Realism and International Politics (pp. 99-122). New York: Routledge.

Waltz, K. N. [1970] (2008a). The Myth of National Interdependence. En Realism and International Politics (pp. 152-165). New York: Routledge.

Waltz, K. N. [1971] (2008c). "Conflict in World Politics". En Realism and International Politics (pp. 19-36). New York: Routledge.

Waltz, K. N. (1979). Theory of International Politics. Reading, Mass.: Addison-Wesley Publishing Company. Tr. cs.: Teoría de la política internacional, Bueno Aires: Grupo Editor Latinoamericano, 1988.

Waltz, K. N. (1986). "Reflections on Theory of International Politics; A Response to my Critics", En Robert O. Keohane (ed.), Neorealism and its Critics (pp. 322-345). Nueva York: Columbia University Press.

Waltz, K. N. (1988). "The Origins of War in Neorealist Theory". Journal of Interdisciplinary History, vol. 18, nº 4, pp. 615-628.

Waltz, K. N. (1990). "Realist Thought and Neorealist Theory". Journal of International Affairs, vol. 44, n 1, pp. 21-37.

Waltz, K. N. (1997). "Evaluating Theories". American Political Science Review, vol. 91, no 4, pp. 913-917.

Waltz, K. N. (2000). "Structural Realism after the Cold War". International Security, vol. $25, \mathrm{n}^{\circ} 1$, pp. $5-41$.

Waltz, K. N. (2003). "Thoughts about Assaying Theories". En Colin Elman y Miriam Fendus Elman (eds.), Progress in International Relations Theory: Appraising the Field (pp. vii-xii). Cambridge: MIT Press.

Waltz, K. N. (2004). "Neorealism: Confusions and Criticism". Journal of Politics and Society, vol. 15, n ${ }^{\circ}$, pp. 2-6.

Wight, M. (1966). "Why is there no International Theory?" En H. Butterfield y M. Wight (eds.), Diplomatic Investigations. Essays in the Theory of International Politics. Cambridge: Harvard University Press.

Zibechi, R. (2009). "Is Brazil creating its own 'backyard' in Latin America?" Upside Down World Latin America, 16 de febrero, disponible en http://upsidedownworld.org/

Zibechi, R. (2013). Brasil potencia: entre la integración regional y el nuevo imperialismo. Lima: Programa Democracia y Transformación Global, Forum Solidaridad Perú. 